\title{
"BLANCO ES UN CONCEPTO BASTANTE DESFASADO...": CANDOMBE AFROURUGUAYO Y CUESTIONAMIENTOS A LA BLANQUITUD EN EL LITORAL ARGENTINO
}

"BRANCO É UM CONCEITO SUFICIENTE DEFASADO ...": CANDOMBE
AFROURUGUAYO E INTERPELAÇÕES À BRANQUITUDE NO LITORAL
ARGENTINO

Julia Broguet

Universidad Nacional de Rosario - UNR Consejo Nacional de Investigaciones Científicas y Técnicas, Argentina

\section{Resumen}

En este artículo me propongo presentar algunos aspectos de una investigación antropológica en curso sobre las relaciones de la práctica del candombe afrouruguayo con los procesos identitarios de sus ejecutantes argentinos. El trabajo de campo ha sido realizado en tres ciudades del sur del Litoral argentino: Paraná, Santa Fe y Rosario, en el periodo que va desde el 2000 hasta el 2015 aproximadamente. Mi interés en este caso es ahondar en las relaciones que se entablan entre la práctica de una manifestación cultural "uruguaya", "negra" y "popular" y las adscripciones étnico-raciales de sus practicantes argentinos.

Palabras claves: Procesos identitários. Candombe afrouruguayo. Litoral argentino.

\section{Resumo}

Neste artigo me proponho apresentar alguns aspetos de uma pesquisa antropológica em curso sobre as relações da pratica do candombe afro uruguaio com os processos identitarios de seus executantes argentinos. O trabalho de campo foi realizado em três cidades do sul do Litoral argentino: Paraná, Santa Fe e Rosário, no período que vá desde o 2000 até o 2015 aproximadamente. Meu interesse neste caso e compreender as relações que se estabelecem 
entre a pratica de uma manifestação cultural "uruguaia", "negra" y "popular" e as identificações étnico-raciais de seus praticantes argentinos.

Palavras-chave: Processos identitários. Candombe afrouruguiao. Litoral argentino.

\section{Introducción}

En este trabajo me propongo presentar algunos aspectos de una investigación antropológica en curso sobre las relaciones de la práctica del candombe afrouruguayo con los procesos identitarios de sus ejecutantes argentinos. Para llevar adelante este objetivo he realizado trabajo de campo en tres ciudades del sur del Litoral argentino: Paraná, Santa Fe y Rosario, en el periodo que va desde el 2000 hasta el 2015 aproximadamente.

Cuando aludo a la práctica del candombe me refiero, inicialmente al candombe afrouruguayo, que se relocaliza en la zona desde finales de la década del '90 y se expande a comienzos del 2000; y a las posteriores recreaciones de un candombe "afrolitoraleño", hacia mediados de este último periodo ${ }^{\mathrm{i}}$. Este análisis comprende el trabajo con jóvenes "blancos"ii, e integrantes de una organización político-cultural de afrodescendientes de la ciudad de Santa Fe. En esta oportunidad me detendré con mayor detalle en el primer estilo, dado que fue el puntapié para el desarrollo del segundo, el cual además cuenta con menos seguidores y espacios de práctica. Así como enfatizaré en los procesos identitarios de los jóvenes, dejando para otra oportunidad contrapuntos y continuidades con los practicantes que actualmente se autoperciben como "afrodescendientes".

Mi interés en este caso es comprender las relaciones de una práctica cultural "uruguaya", "negra” y "popular" con los procesos identitarios de sus practicantes argentinos que, respecto a la misma, podrían identificarse como "blancos" y "de clase media"iii. De allí que, en primer lugar, creo necesario explicitar qué entiendo por "raza", en tanto categoría que adquiere relevancia en la temática investigada ${ }^{\mathrm{iv}}$.

Parto de la consideración de Briones (2005) acerca de la raza como una construcción sociohistórica y, al mismo tiempo, categoría de análisis social que da cuenta de formas diferenciadas de marcación de alteridad. 
En tal sentido, sería más preciso hablar de prácticas de racialización, a fin de evitar darle una entidad definida y conclusiva a lo que es su producto ("lo negro"). Esta expresión describe el proceso a través del cual se le adjudican a ciertas marcas corporales implicancias sociales, morales, culturales, políticas. Al referirme a una persona (o práctica) racializada, intento evitar el uso reificado de categorías como "blanco" o "negro", como si fueran "formas objetivas de agrupamiento humano" o "cualidades tipológicas de grupos concretos" (BRIONES, 2005, p.65). Pretendo subrayar, y esto es un tema sobre el cual volveré a lo largo del trabajo, que blanco y negro existen en relación, son situacionales y precisan ser definidos (y deconstruidos) en contextos específicos (SANSONE, 2003). Por eso me parecen sugerentes algunas observaciones que hacen los estudios "de la blanquedad" (MORENO FIGUEROA en CUNIN, 2010). En la medida en que permiten exponer aquellas identidades que, por efecto de relaciones de poder y resistencia desiguales, operan como "paradigmas implícitos normalizados" - y normalizadores - (RESTREPO, 2007, p.29). De este modo, la blanquedad no está necesariamente asociada al "cuerpo blanco" sino a un "espacio de privilegio" que se ocupa de manera más o menos "fugaz" y siempre "relacional" (MORENO FIGUEROA en CUNIN, 2010, p.79).

En segundo lugar, me interesa pensar estas dimensiones raciales en la articulación entre raza y nación. Es decir, cómo las identificaciones raciales fueron parte de los procesos de formación de los Estados latinoamericanos y de las identidades nacionales (WADE, 2002). La imagen del mestizaje como síntesis de tres "razas" (indígena, negra y europea) fue adoptada en países como México, Colombia o Brasil. En el caso argentino, el proceso de formación del estado-nación se produjo con los intentos de sus elites intelectuales de construir una narrativa de nación blanco-europea que excluyó lo no-blanco (indígena y afro) como su alter ego. De este modo, y atendiendo a lo que fue emergiendo en el propio trabajo de campo, este eje raza-nación lo abordaré teniendo en consideración cómo la blanquitud/negritud -así como todo aquello que no entra en esa clasificación binomial- también es parte de una "experiencia racializada cotidiana" (CUNIN, 2010, p.12) que "se da sobre y dentro de los cuerpos de las personas, y dentro de las familias, tanto como dentro de la nación" (WADE en CUNIN, 2010, p.62) ${ }^{\mathrm{v}}$. Pese a que, por lo general, estos efectos prácticos no sean reconocidos, 
BLANCO ES UN CONCEPTO BASTANTE DESFASADO...”: CANDOMBE AFROURUGUAYO Y CUESTIONAMIENTOS A LA BLANQUITUD EN EL LITORAL ARGENTINO

pues se suele afirmar que en Argentina "no hay negros" y en consecuencia el racismo no sería una problemática que nos ataña como sociedad.

Organizaré el trabajo en cuatro partes y unas breves conclusiones. En la primera, situaré al candombe como un género con diferentes versiones de uno y otro lado del Rio de la Plata, para luego identificar sus disimiles trayectorias tomando como referencia las ciudades de Buenos Aires y Montevideo. Estas diferencias pueden asociarse a las particulares formaciones nacionales de alteridad (BRIONES, 2008) de Argentina y Uruguay, que produjeron en su interior lugares diferentes para la población afrodescendiente ${ }^{\mathrm{vi}}$. A partir de allí, ubicaré cómo se relocaliza el candombe afrouruguayo en la Argentina de las últimas décadas, haciendo énfasis en su circulación como parte de un "circuito afro"vii (por el que se desplaza un público perteneciente a las capas medias y en el cual se encarnan y producen sentidos de "lo negro"); y buscando describir singularidades del modo de circulación del candombe en ciudades del Litoral argentino.

En la segunda, y desplegando puntos anticipados en el apartado previo, describiré el particular funcionamiento de categorías raciales en nuestro país, para así comprender su incidencia en las modalidades de apropiación del candombe afrouruguayo en la zona en análisis.

En la tercera, situaré dimensiones históricas y subjetivas que al momento de expansión del candombe afrouruguayo trazaron el rumbo de sus modalidades de apropiación. Estas propician y movilizan las que he llamado experiencias de negritud, sobre las cuales profundizaré aquí.

En la cuarta y última sección, retomando lo desarrollado anteriormente, ahondaré en un caso de identificación con categorías de negritud. Así, quiero exponer cómo las modalidades de apropiación locales de esta práctica de cultura "negra" y "ajena" interroga en términos raciales, de maneras múltiples e incluso contradictorias, a sus practicantes argentinos "blancos". Estos procesos identitarios advierten sobre la necesidad teórica de distinguir cultura-etnicidad-identidad para evitar relaciones de equivalencias necesarias entre estos términos. Así como dan cuenta de la maleabilidad de las categorizaciones raciales. En las conclusiones recogeré parte de este recorrido para sugerir algunos interrogantes que éste plantea. 


\section{Visibilidades/invisibilidades del candombe en el Rio de la Plata}

Según Frigerio y Lamborghini (2009), las prácticas musicales (y dancísticas) de los esclavos traídos a Buenos Aires y Montevideo durante la época colonial reciben, a partir del siglo XIX, el nombre de candombe. Como una práctica cultural asociada a una minoría étnico-racial, su desarrollo se da de forma distintiva en ambas capitales ${ }^{\text {viii }}$.

Así, mientras que en la Buenos Aires del siglo XX el candombe porteño va progresivamente saliendo del espacio público (en consonancia con el afianzamiento de la idea de "desaparición" de la población negra de la nación), para refugiarse en clubes y luego en casas de familia, quedando circunscripto al ámbito endogrupal (CIRIO, 2007) ${ }^{\mathrm{ix}}$; el candombe montevideano, por el contrario, gana espacios públicos (REID ANDREWS, 2006), superando barreras étnico- raciales, de clase social, de género y de nacionalidad (FRIGERIO y LAMBORGHINI, 2012).

Como lo señalan Abril Trigo (1993) y Reid Andrews (2006), en Montevideo el candombe se extendió a la población en general, convirtiéndose poco a poco en un "ritmo nacional” que circuló más allá de los barrios con mayor población afrodescendiente ${ }^{\mathrm{x}}$. En cambio, como parte de los esfuerzos de elites políticas e intelectuales por consolidar a Argentina como una nación moderna (como equivalente de "blanca y europea"), se tendió a “desaparecer" de manera paulatina a la población afrodescendiente y a sus prácticas culturales (REID ANDREWS, 1989). Además, investigaciones más recientes señalan cómo esta narrativa blanqueada se impuso a "los complejos procesos de mestizaje que se dieron en las distintas provincias del territorio nacional” (FERNÁNDEZ BRAVO, 2016, p.164). En tal sentido, muy lejos de la dinámica que en Uruguay volvió al candombe un "ritmo nacional", cuando en la década del '40 comenzó a formarse en Argentina un "repertorio folklórico nacional" compuesto por diferentes "músicas y danzas", éste se produjo en consonancia con una ideología que "valorizaba fundamentalmente la raigambre europea" (CITRO, BROGUET, RODRÍGUEZ, TORRES AGÜERO en prensa). Así, el musicólogo argentino Carlos Vega $(1944,1952)$ referente principal de la documentación de estas “músicas y danzas folklóricas”, excluyó de sus estudios a los grupos indígenas y afrodescendientes que existían 
BLANCO ES UN CONCEPTO BASTANTE DESFASADO...”: CANDOMBE AFROURUGUAYO Y CUESTIONAMIENTOS A LA BLANQUITUD EN EL LITORAL ARGENTINO

en el país, y consideró que solamente un par de géneros folklóricos de la región del noroeste andino contenían raíces "indígenas" y ninguno "afro" (CITRO, BROGUET, RODRÍGUEZ, TORRES AGÜERO en prensa).

De modo tal que, décadas más tarde, hacia los años '90, momento de expansión en Argentina de la práctica del candombe afrouruguayo entre sectores juveniles "blancos" y de "clase media", el candombe argentino no daba (aún) ningún indicio de su existencia y permanencia actual. Si aparecía, era como parte de una estética escolar que escenificaba un pasado ya "desaparecido" (el mismo al que se había empujado a los propios "negros argentinos"), mostrando un candombe altamente exotizado, "alegre y colorido", en los actos patrios anuales (OCORO LOANGO, 2011).

\section{El arribo del candombe afrouruguayo a Argentina y su inserción en un "circuito afro"}

De acuerdo a investigaciones realizadas por Frigerio y Lamborghini (2012) y Parody (2014) el candombe montevideano llega a la ciudad de Buenos Aires a fines de los años '70. Traído por migrantes afrouruguayos, su territorialización se produce, en primer lugar, en el barrio de San Telmo. Desde allí, el grupo migrante comienza a reproducir, a principios del '80, las "llamadas"xi de forma espontánea en los días feriados, tal como se realizan en Montevideo. Hacía fines de esa década, una segunda generación de negros jóvenes, apelando a la memoria de la presencia negra en Argentina, se ocupa de difundir y enseñar su arte a sus congéneres blancos argentinos.

Según Frigerio y Lamborghini (2012), hacia el año 2005, el candombe se expande por diferentes barrios porteños, y por el resto del país; asunto que no se ajusta a lo relevado en las ciudades de Santa Fe, Paraná y Rosario. En estos centros urbanos del Litoral, la práctica del candombe afrouruguayo comienza hacia fines de los '90, tiene un momento clave en el contexto posterior a la crisis socio-económica del 2001, cuando se afirma como parte de un circuito que reúne diferentes performances "afro" (arribadas al país a través de migrantes afrodescendientes de países del Cono Sur); y se formaliza a lo largo de la primera década del siglo $\mathrm{XXI}^{\mathrm{xii}}$.

Este proceso se desarrolla paralelamente a una revalorización de la cultura afroargentina y a una serie de cuestionamientos en torno a "lo negro" como un componente "invisibilizado" del pasado y el presente de la nación ${ }^{x i i i}$. Lo cual instala entre sus practicantes argentinos una 
serie de interrogantes -históricos, políticos, existenciales- sobre los modos de representación dominantes de la argentinidad como "blanca y europea" (en especial en lo que refiere a sus valoraciones racistas y clasistas). Vía la incorporación y ejecución (e incluso recreación) de diversas prácticas culturales consideradas "afro", estos sectores comienzan a reivindicar "lo negro" en tanto signo de lo "negado, oculto, despreciado y no-occidental"xiv.

La práctica del candombe afrouruguayo se formaliza a lo largo de la primera década del siglo XXI en estas tres ciudades. De la mano de un interés por adentrarse en sus códigos específicos y vivenciarlo como performance total -ni estrictamente musical, ni medio para un fin- sino como práctica que reunía música, experiencia corporal, historia, lazo social y vida cotidiana (BROGUET, PICECH, RODRÍGUEZ, 2012 y 2014).

\section{Algunas singularidades locales}

Para cerrar este apartado mencionaré singularidades del candombe afrouruguayo como práctica juvenil en la zona, respecto a otros centros urbanos en los que también fue investigada (ESPINOSA, 2016; LAMBORGHINI, 2015).

Primero, a diferencia de lo relevado en Salta y Jujuy por Espinosa (2016) - quien registra memorias en torno a "barrios negros" entre fines de siglo XVIII y comienzos del XIX, pero no de sus prácticas culturales -, en esta región hay dos antecedentes históricos de poblaciones negras locales que ejecutaban "candombes" "xv . En ambos casos, esos antecedentes no tuvieron continuidad al presente y hoy funcionan para sus practicantes locales como parte de un "legado cultural africano" propio de la zona ${ }^{x v i}$. Estas memorias, escritas y orales, comienzan a circular con más fuerza luego de la crisis del 2001, en particular en Santa Fe y Paraná, y a partir de ellas se desarrollan eventos que convocan y nuclean a agrupaciones de candombe de estas dos ciudades. En particular, el llamado "Contrafestejo", una "llamada" que se realiza desde el año 2002 en la ciudad de Paraná, los días previos al 12 de octubre. De hecho, aunque este evento empezó a coincidir con otra fecha anual importante para los practicantes argentinos, como son los "Encuentros de CandombeS" -que comienzan en 2007 y reúnen a muchas agrupaciones candomberas del país-, los organizadores locales resolvieron no cambiar la fecha.

Revista RBBA $\mid$ Revista Binacional Brasil Argentina 

LITORAL ARGENTINO

Segundo, mencioné la presencia de la Casa de la Cultura Indoafromericana como organización político-cultural de afrodescendientes argentinos, y es necesario subrayar su incidencia en los procesos locales vinculados al candombe. A su vez, y en contraste con lo que sucede en Buenos Aires, en ninguna de las tres ciudades trabajadas se localiza una población afrouruguaya migrante importante numéricamente. De allí que, como grupo migrante, tuvo menos incidencia en la difusión del candombe en la zona respecto a otras dinámicas de contacto "directo" con candomberos afrouruguayos de Montevideo. En este sentido, en el ámbito local no aparece, como sí se releva en la ciudad de Buenos Aires (FRIGERIO y LAMBORGHINI, 2012, p.96), una urgencia por "justificar su práctica en condiciones que no sean de inferioridad" lo que identificamos vinculado a la cotidianeidad en la interacción con el grupo migrante (BROGUET, PICECH y RODRÍGUEZ, 2012 y 2014)

Tercero, en estas ciudades, el candombe afrouruguayo, como género especifico, no ha formado parte de las políticas culturales de organismos estatales, salvo cuando se ha visto incluido en talleres de "percusión” junto a muchos otros géneros. Tampoco hubo intentos de regular la actividad de las agrupaciones de candombe, como si refiere Lamborghini (2015) para Buenos Aires. Ni existe ninguna legislación que reconozca institucionalmente y promueva su difusión, como sí sucede desde 2013 en esta misma ciudad. Los vínculos con organismos del Estado han sido bastante ocasionales e irregulares, con el fin de gestionar algún permiso para espectáculos en la vía pública, algún recurso para la organización de eventos (como escenarios y/o baños químicos) o por algún encuentro con funcionarios policiales a causa de denuncias de vecinos por "ruidos molestos".

Cuarto, y aunque sea un punto sobre el cual no ahondaré aquí (BROGUET, 2008; BROGUET, PICECH y RODRÍGUEZ, 2012; BROGUET, 2017), es necesario mencionar que en el intercambio entre practicantes de Paraná y Santa Fe se produjo un proceso inédito de recreación de un candombe "propio". Hacia mediados del 2000, un músico paranaense, comienza un trabajo de recreación de unos "candombes del Litoral" junto a músicos santafesinos e integrantes de la Casa. Inicialmente se circunscribe al "universo sonoro del Barrio del Tambor de Paraná en el siglo XIX"xvii, para luego ampliarse a una investigación sobre música "afrolitoraleña" que se extiende a la región. 


\section{Blanquitud/negritud en Argentina}

"[Son] nuestras fisuras y clivajes interiores y los materiales heterogéneos y discontinuos con que construimos nuestro mundo (...) las puertas y ventanas por las cuales entramos en comunicación con mundos ajenos" (SEGATO, 2005, p.260).

Para comprender cómo las modalidades de apropiación del candombe afrouruguayo movilizan lo que llamo experiencias de negritud, creo necesario comenzar por subrayar la "incomodidad" registrada por muchos de los practicantes argentinos, o la "incoherencia" señalada por algunos espectadores de estas manifestaciones en el ámbito local (la cual además utilizo como coordenada de análisis $)^{\text {xviii }}$, en torno a realizar una práctica cultural "negra" siendo "blancos". Quizás el formular la pregunta inversa permita desandar esta aparente contradicción: ¿Por qué un "argentino, blanco y clase media" no podría practicar una manifestación “uruguaya, negra y popular”? ¿Será porque se asume una equivalencia entre práctica cultural y etnicidad negra por la cual solo los "negros" podrían hacer "naturalmente" candombe afrouruguayo? ¿Qué nos dicen estas asociaciones sobre los modos locales de categorizar socialmente "lo blanco" y "lo negro"?

Primero, cabe aclarar que la identificación como "blancos" de los practicantes argentinos se explicita sólo a partir de la ejecución de una práctica de cultura considerada "negra". Hasta entonces, la blanquedad no es algo que se problematice abiertamente. En todo caso, se asume como parte de la norma racial implícita que produjo la representación más difundida de la "argentinidad". Lo que intentaré exponer es cómo esta identificación inicial muta con el tiempo de inmersión en la práctica, exponiendo conflictos irresueltos respecto a la imagen de Argentina como nación "blanca y europea".

Para comenzar creo necesario exponer brevemente lo que Frigerio (2006) analizó como el funcionamiento de formas de categorización racial en Buenos Aires. Su planteo es que la construcción de la negritud y de la blanquitud ${ }^{\mathrm{xix}}$

se [basa] en el interjuego entre una modalidad hiperrestrictiva (la

negritud) y una hiperinclusiva (la blanquitud), que [terminó]

Revista RBBA $\mid$ Revista Binacional Brasil Argentina 
Así, la blanquitud y la negritud (como su extremo opuesto) se constituyen (y excluyen) mutuamente, como parte de lo que Geler (2016, p. 78) -quien retoma a Frigerio (2006)describe como un "sistema de categorización racial binomial" que "imposibilita lo mestizo", provocando un arco de experiencias intermedias que son "absorbidas" por la blanquitud" Así, categoriza al contrapunto de esta blanquitud hiperinclusiva como "negritud racial"xxi. La lógica que produce esta negritud hiperrestrictiva como "epitome de lo racial” es la que, según Briones (2008, p. 24), “lleva simultáneamente a negar la existencia del racismo en el país y a interiorizar las líneas de color"xxii.

Esta negritud racial se articula con la existencia de "otro tipo de negros" (GELER, 2016), que sí forman parte de lo connacional. Se trata de una forma de categorización por clase social, que, sin depender exclusivamente de marcadores visuales de raza, se refuerza en ellos. Como advierte Frigerio (2006, p. 11) las tonalidades de piel más -o menos- oscuras del histórico "cabecita negra", y del actual "negro villero", "proviene del mestizaje ancestral, no solo con indígenas sino también con la numerosa población negra que existió en varias provincias en el siglo XVIII". Así, estos motes, dirigidos usualmente a sectores mestizos de bajos ingresos, serían la síntesis de una negritud connacional que alude a una "forma de ser" inculta, inmoral, criminal- asociada al mundo popular (GELER, 2016, p. 75). Por todo esto, la autora la designa como "negritud popular", sin dejar de reconocer los múltiples cruces y mutuos reforzamientos entre ambas formas de categorización social (negritud racial y popular).

De cara a los matices que presenta el funcionamiento de categorías de blanquitud y negritud en Argentina, es evidente que ninguna identidad trabaja como un monolito "de puro poder o de pura resistencia" (RESTREPO, 2007, p.28). De allí que, en determinadas circunstancias históricas, sus cabos "sueltos" pueden (o no), articularse entre síxxiii. Por lo que (y en parte respondiendo a aquellas acusaciones de "snobismo" que algunos observadores externos les hacen a los practicantes argentinos de candombe afrouruguayo), las identificaciones con categorías de negritud analizadas en este contexto de investigación no surgen por "generación espontánea", ni son pura fascinación y exotización. Devienen también 
de prácticas, memorias, vínculos sociales (institucionales, familiares) de los practicantes locales de candombe afrouruguayo que son previos a su acercamiento al mismo, y que reúnen elementos heterogéneos, ambiguos e incluso contradictorios entre sí. Son experiencias que en general quedan excluidas por esta lógica de categorización racial binomial, según la cual Argentina es "blanca" y ya "no hay negros negros", no está bien visto que los "blancos" hagan "cosas de negros" y, los "negros" son llamados a "blanquearse".

Intentaré exponer cómo estos cuestionamientos a las formas de categorización racial locales se producen con la inmersión de estos practicantes en un "modo de hacer", como lo es el candombe, que moviliza diferentes experiencias de negritud ${ }^{x x i v}$.

\section{Modalidades de apropiación y experiencias de negritud}

En los primeros años de difusión del candombe afrouruguayo, muchos de sus practicantes (sobre todo estudiantes universitarios o terciarios de carreras humanísticas y/o artísticas que habitan/recorren las zonas céntricas de estas localidades), nacidos entre mediados de 1970 y 1980, compartían aspectos que pueden entenderse como un núcleo problemático generacional. Primero, el enfrentar un discurso instalado sobre la supuesta apatía política de la juventud. Segundo, sobrevolaba un fantasma de que la generación precedente "lo había dado y dicho todo". Y tercero, la necesidad de indagar en un pasado que en cierta medida aparecía "cortado", "negado", "silenciado", vinculado al contexto político de la década del '90 y sus políticas de memoria respecto al último periodo dictatorial, que cubrían lo sucedido con un manto de falsa armonía (ALUCÍN y BIASATTI, 2015). Es así que aparece una diferenciación generacional hecha por algunos de los practicantes, entre la preocupación de una generación previa a ellos, por un “decir" en la música (por el "contenido" de las letras) frente al interés por los "modos de hacer". Esto es, una necesidad de "poner el cuerpo" (VVAA 2014) hacer de él un terreno de experimentación expresiva, ocupando el espacio público y explorando lenguajes no exclusivamente textuales para comunicar. Así, la adquisición de destrezas físicas y de estados emocionales particulares a partir del aprendizaje del baile y los toques específicos de cada tambor de candombe, como de la comunicación necesaria entre ellos; tanto como los sentidos racializados (siendo central el 

LITORAL ARGENTINO

de "resistencia") que condensa su práctica en relación a la historia de la población afrouruguaya $^{\mathrm{xxv}}$, jugaron un papel central (BROGUET, PICECH y RODRÍGUEZ, 2012) en la voluntad de reponer y encarnar esos cuerpos “desaparecidos” de la escena pública ${ }^{\mathrm{xxvi}}$.

Al caracterizar la práctica del candombe como un "modo de hacer", me interesa subrayar el lugar que ocupa la experiencia corporal, sensorial y afectiva, en complejas articulaciones con esos sentidos otorgados a la práctica (que se transforman y actualizan en un diálogo constante). En este sentido, en las ciudades trabajadas se producen fases o modos similares en torno a la llegada del candombe, que en otro trabajo (BROGUET, 2012) caracterizamos en dos etapas. La primera abarca el período en que el candombe es escuchado y ejecutado a través de la canción rioplatense ${ }^{\mathrm{xxvii}}$, hasta el momento de aparición del tambor de candombe afrouruguayo, en el que comienza a ser tocado y bailado "en la calle". Estas etapas están marcadas por diferentes modos de circulación, ejecución y experiencias prácticas/corporales del candombe. Del mismo modo en que están mediadas por modos de transnacionalización particulares, que le imprimieron una dinámica a su desarrollo y expansión. Los aprendizajes del candombe afrouruguayo como práctica comunitaria "en la calle" se produjeron de persona a persona, a través de viajes particulares, de migraciones, de intercambios culturales múltiples en coordenadas étnico-raciales, nacionales, de clase y género. En tal sentido, este proceso se adecuaría a lo que Frigerio (2002) describió como una “transnacionalización desde abajo". Mientras que el candombe escuchado y ejecutado en la canción rioplatense, responde en general a un tipo de transnacionalización "desde arriba", promovida mayormente por industrias culturales como parte del fomento al consumo de ciertos bienes simbólicos y regulado por dinámicas propias de la escena artístico-musical. Si bien esta es una generalización y sería incorrecto desconocer que estos procesos se entrecruzan de múltiples maneras, su distinción es eficaz para comprender la dinámica "cuerpo a cuerpo" que supuso inicialmente la inmersión en el candombe para los practicantes del Litoral argentino durante la década del ' 90.

Lejos de reducirse a un mero objeto de consumo, a una "moda" o a un "robo" o "rapto" cultural $^{\text {xxviii }}$, sería más justo ubicarlo como parte de lo que podríamos llamar un interjuego cultural que produce distintas modalidades de apropiación -múltiples, relacionales y transformadoras (ROCKWELL, 2005, p.33)- que tensionan las adscripciones étnico-raciales, nacionales, de género y clase de los sujetos involucrados. 
A través de estas modalidades de apropiación del candombe afrouruguayo en Argentina, se producen distintas experiencias de negritud. Esta propuesta intenta situar el carácter vívido y creativo de este interjuego. Cómo el acercamiento de los practicantes argentinos a esta práctica cultural suscita, moviliza (y transforma) prácticas, sentidos y experiencias (previas y simultáneas) que son parte de un régimen dominante de representación ligado a la expansión colonial europea - que luego se articulará en cada

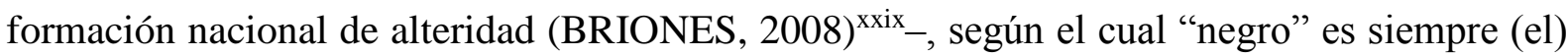
“otro" (HALL, 2010; SEGATO, 2007). Así, a partir de la observación de estas modalidades de apropiación y experiencias de negritud en el Litoral argentino, he notado un desplazamiento: desde una mirada exotizante (y quizás verdaderamente "ajena" en tanto ancla en representaciones estereotipantes de la negritud) a un involucramiento que redefine las propias adscripciones sociales de sus practicantes ( $\sin$ dejar por eso de reconocer el papel que los estereotipos juegan en la incorporación del candombe, pero no reduciéndolo a ese fenómeno), en un movimiento que va de la representación a la experiencia y viceversa.

Con la propuesta de experiencias de negritud la intención es poder subrayar cómo esos legados coloniales ligados a una diáspora forzada de africanos negros esclavizados hacia América inciden en el presente de quienes hoy se autoreconocen como "afroargentinos" (y desde esa construcción identitaria generan reclamos y reivindicaciones), como en el de los otros grupos humanos que constituyen a la sociedad argentina en su conjunto (Subrayado propio. CUNIN, 2010; RESTREPO, 2003) ${ }^{\mathrm{xxx}}$.

En el cotidiano, estas experiencias se ligan a modos de hacer y sentidos asociados a la negritud que se ponen en juego en la práctica del candombe afrouruguayo. Aunque admiten puntos similares, pues comparten un mismo basamento histórico, también comprenden diferencias profundas. Primero, porque devienen de la experiencia de "dispersión y fragmentación" que supuso la diáspora forzada hacia el continente americano ${ }^{\mathrm{xxxi}}$. Segundo, porque se inscriben de formas específicas en cada formación nacional de alteridad (BRIONES, 2008), en este caso es preciso considerar dos contextos nacionales en juego (Uruguay y Argentina). De esto se deduce que estas experiencias comprenden y articulan modos singulares de categorizar la negritud. 

LITORAL ARGENTINO

Para poder abordar estos procesos consideraré dos tipos de experiencias de negritud en diálogo entre sí que son suscitadas y movilizadas por las modalidades de apropiación del candombe afrouruguayo en el Litoral argentino. El primer tipo refiere a cómo se inscribe la experiencia histórica de la población africana y afrodescendiente en los modos de hacer (en especial sus dimensiones expresivas: el toque de tambores y el baile) propios del candombe afrouruguayo. En otro trabajo profundicé en aspectos de esta dimensión (BROGUET 2014). Para sus practicantes argentinos "hacer candombe" supone "hacer cuerpo" esa historia relatada por su performance. Las gestualidades, movimientos, significaciones y estereotipos asociados al toque de tambores y al baile del candombe afrouruguayo tienen un carácter performativo suscitando este tipo de experiencias ${ }^{\mathrm{xxxii}}$. Estos modos de hacer se fueron constituyendo y transformando en un contexto moderno-colonial de relaciones de poder desiguales que tuvieron efectos estructurantes en la configuración de "cuerpos, identidades, sexualidades, espacios y modos de representar" lo mismo y lo otro (RESTREPO, 2003, p. 97).

El segundo tipo alude a experiencias cotidianas con lo negro ${ }^{\text {xxiii }}$ que son previas al acercamiento de los practicantes del Litoral argentino al candombe afrouruguayo de allí que son movilizadas con su inmersión al mismo. Se trata de modos de comportamiento social, prácticas culturales locales (como la cumbia, el carnaval o las batucadas), categorizaciones por color de piel, modos de narrar las memorias familiares, entre otros elementos, en los cuales "lo negro" aparece como un atributo con carácter valorativo y/o explicativo. Pueden haberse vivido como propias o siendo testigos de las mismas. Además, estas experiencias ponen en juego otras dimensiones que se articulan a lo étnico-racial, como la clase social (BROGUET, en prensa) o los ordenamientos espaciales (BROGUET, 2016). Este segundo tipo de experiencias de negritud responde a aquello que Frigerio (2006) caracterizó como uno de los aspectos que permitió construir blanquitud en nuestro país: un desplazamiento de lo racial a lo social ${ }^{\text {xxxiv }}$.

Son ejemplos de este segundo orden de experiencias de negritud -las cuales se hacen legibles en una nueva clave con la inmersión en los modos de hacer candombe afrouruguayo, los señalamientos del color de piel "oscuro" que realizan algunos practicantes, como indicio de "lo negro" -en sentido amplio de no-europeo- en memorias familiares "blanqueadas", punto que retomaré en el próximo y último apartado. En éstos se registra cómo en la trayectoria de una misma persona pueden reconocerse una multitud de identificaciones que 
dan cuenta del carácter radicalmente "histórico, situado y cambiante" de las categorías raciales en Argentina (GELER, 2016) las cuales no pueden ser comprendidas desde una pura blanquitud y/o negritud pues, bajo este punto de vista, aparecen (y son vividas) como “contradictorias"-como mencioné, un "blanco" no podría hacer "cosas de negros"- ${ }^{\mathrm{xxx}}$.

\section{La "oscuridad" en memorias familiares "blanqueadas"}

En otro trabajo (BROGUET en prensa) indagué en las relaciones posibles entre la práctica del candombe afrouruguayo (los sentidos de negritud racial, pero también popular, que vehiculiza) y los cuestionamientos que algunos ejecutantes del Litoral argentino hacían a sus propias memorias de familias de "clase media", "blanqueadas", en tanto reflejo distorsionado de la representación de Argentina como nación blanco-europea ${ }^{\mathrm{xxxvi}}$. Esta interpelación se producía a partir de los señalamientos que los practicantes realizaban del color de piel "oscuro" (propio o de algún familiar cercano) como un indicio de no-blancura, de no-europeidad, de la presencia de antepasados negros e indígenas omitidos de las narrativas familiares, todo lo cual entraba en contradicción con la idea de blanquitud/europeidad como síntesis de la nación.

Además, estos señalamientos en general venían acompañados por relatos de episodios de la infancia y/o adolescencia, en contextos institucionales (escolares sobre todo), familiares y barriales, en los cuales ese color de piel "oscuro" - el hecho de que alguien lo señalara como un rasgo visible - había tenido un papel relevante (pudiendo ser objeto de motes peyorativos, como "negra esclava" o "chupetín de bleque" o de formas de distinción que en el cotidiano familiar y/o barrial podían matizarse con apelativos cariñosos tanto como recuperar la carga negativa asociada al "ser negro").

Al generarse este registro en torno a esas experiencias de negritud que los habían involucrado íntimamente, también sucedía que la idea de blanquitud que ellos refrendaban para sí mismos (en el contraste con la ejecución de una práctica racializada "negra"), aparecía algo más desdibujada. Lo que cambiaba era su posición. Es decir, respecto a quien $-\mathrm{o}$ a quienes- y en qué situaciones intersubjetivas, ellos estaban identificándose o diferenciándose como "blancos" o "no-tan-blancos".

\begin{tabular}{l|l} 
Revista RBBA & Revista Binacional Brasil Argentina
\end{tabular} 

LITORAL ARGENTINO

Así, lo que comencé a identificar en los relatos de experiencias previas a su acercamiento a esta manifestación, fueron interacciones cotidianas que habían estado marcadas por una dimensión racial con un papel activo en la elaboración de clasificaciones sociales. A su vez, observé que la clave en la que hoy se interpretaban y problematizaban estas experiencias personales guardaba relación con la inmersión de estos practicantes en una práctica cultural racializada (que movilizaba experiencias que "ennegrecían" al cuerpo), la cual les habilitaba a ir nombrando una problemática de otro modo implícita en nuestro país como lo es el racismo- y no por ello con menos efectos sociales

De este modo, la posibilidad de ubicar estas experiencias personales en clave racial les permitió ir poniendo en duda la propia blanquitud como construcción social. Es decir, si respecto a los afrouruguayos que ejecutan candombe -en tanto "representantes legítimos"ellos eran designados o aceptaban ser designados como "blancos", esta evidencia se opacaba cuando emergían experiencias previas en las que su propia "negritud" -en un sentido amplio de no-europeidad- había sido señalada, friccionando el sentido común de la "normalidad" argentina, incluso a nivel de las tramas familiares ${ }^{\text {xxxvii }}$.

Este cuestionamiento a la exclusión de mujeres y hombres indígenas y/o afro del nosotros nacional/familiar, re-sitúa a estos practicantes como sujetos de una historia que ya no es "la de otros" sino la "propia". Como dejé sugerido en el trabajo referido, estos procesos que se producen entre practicantes del Litoral argentino, de incorporación de "lo negro" (afro e indígena) a memorias familiares blanqueadas, pueden entenderse como un movimiento que "des-otreriza" la diferencia - entendiendo aquí la otrerización como estrategia que establece una negatividad constitutiva y des-marca (o "emblanquece") a la mismidad (RESTREPO, 2013).

\section{Nombrar la raza en Argentina}

En este trabajo quise exponer los nudos centrales de una investigación en proceso. Me refiero a ese lento proceso investigativo relacionado al abandono de algunas certidumbres, así como al riesgo de establecer otras relaciones entre elementos que hasta entonces no habíamos considerado lo suficiente. Me planteé presentar estos nudos a través de cuatro apartados interconectados, que permitieran situar en clave actual, pero también histórica, la problemática más amplia en la que se inscriben. Estos apartados fueron, primero, una ajustada 
síntesis de los diferentes estatus de "lo negro" en la conformación de las distintas "formaciones nacionales de alteridad" en Uruguay y Argentina, a fin de exponer cómo esto tuvo un papel significativo en la expansión del candombe afrouruguayo hacia fines de la década del '90 y comienzos del 2000 en el país, así como en su incorporación a un "circuito afro" y en las dinámicas particulares de apropiación del candombe afrouruguayo producidas en el Litoral argentino. En segundo lugar, retomando la tesis de Frigerio (2006) sobre el funcionamiento de categorías raciales en Argentina, luego ampliada por Geler (2016), propuse que las modalidades de apropiación del candombe afrouruguayo en Argentina también están sujetas a este sistema de categorización que crea dos tipos de negritudes (racial y popular). Estas modalidades, propician y movilizan diferentes experiencias de negritud, descriptas en el tercer apartado, poniendo en dialogo distintos niveles de análisis, temporalidades, prácticas y sentidos que se les asocian. Y cuarto, describí un caso de identificación con categorías de negritud que se produce entre algunos practicantes a través de los señalamientos que hacen de la "oscuridad" de la piel, un indicio de negritud -en sentido amplio de no-europeidad- en memorias familiares "blanqueadas".

Las experiencias de negritud suscitadas y movilizadas por la práctica del candombe afrouruguayo habilitan así a nombrar pasajes de la vida íntima y/o colectiva de los practicantes argentinos, vinculados a prácticas de racialización producidas en su cotidiano, hasta entonces ocluidos, y por lo mismo, ininteligibles ${ }^{\mathrm{xxx} \text { iii }}$. Como parte del mismo ejercicio de "marginación" - en el doble sentido, de delimitar y, en simultaneo, dejar afuera- que funda las identidades - y su "exceso", ese "algo más" necesariamente excluido y desjerarquizado (HALL, 2011, p. 19)-, la representación de una argentinidad europeizada se muestra incapaz de hacer lugar a la multitud conflictiva de cuerpos e historias presentes y vivas en este territorio nacional.

Es decir, la blanquitud es una posición a la cual somos convocados por el ideal normativo regulatorio de una narrativa nacional argentina blanco-europea, pero esa identificación nunca se produce en su completitud, "de una vez y para siempre", como bien sugiere Hall (2011, p. 32). Los procesos identitarios de los cuales quise dar cuenta en este trabajo no intentan "recuperar" una identidad "negra originaria", si por eso se entiende volver a algún lugar concreto donde ésta se extravió y permaneció igual a sí misma, como si el 
devenir histórico permitiera que esto sea posible. En todo caso, quise exponer cómo esos procesos se sitúan en un interjuego de miradas estereotipantes y "ajenas" a un involucramiento íntimo que pone en riesgo aquellas certezas que otorgan los estereotipos, se desplazan desde representaciones acerca de un "otro" racial hacia lo "otro" racial como parte constitutiva de ellos mismos, conjugan diferentes dimensiones sociales (nacional/familiar) y formaciones nacionales de alteridad, en las cuales "lo negro" tuvo y tiene un papel diferencial.

A partir de lo expuesto, creo que la explicitación del racismo en Argentina, a través de la relectura de experiencias personales y grupales bajo su lupa (la cual en este caso se produce a través de la inmersión en una práctica cultural racializada) sigue apareciendo como un primer (e ineludible) paso para dejar expuesto, comprender -y así afrontar- este fenómeno en nuestro contexto.

Asimismo, en estos procesos que cuestionan de distintas maneras "lo blanco" como entidad cegada y enrarecida - pero también "lo negro" en tanto se establece en relación forzosa con aquel, exigiendo a veces "pruebas" de negritud ${ }^{\mathrm{xxxix}}$-, queda sugerida una pregunta latente, que nos empuja a lo incierto cuando nos interroga acerca de en qué podríamos convertirnos si abandonamos las garantías tranquilizadoras que otorgan las categorizaciones raciales. Esto es si, finalmente, podemos ir "más allá"xl de las aparentes certezas que puedan dar la blanquitud y la negritud.

\section{Referencias}

ADAMOVSKY, E. El color de la nación argentina. Jahrbuch für Geschichte Lateinamerikas, $\mathrm{n}^{\circ} .49$, pp. 343-364, 2012.

ALUCÍN, S. y BIASATTI, S. Cruce de tesis. Rosario: UNR Editora, 2015, p.456.

BRIONES, C (comp.). Mestizaje y blanqueamiento como coordenadas de aboriginalidad y nación en Argentina. Runa XXIII, pp. 61-88, 2005.

- Cartografías argentinas: políticas indigenistas y formaciones provinciales de alteridad. Buenos Aires: Antropofagia, 2008, p.287

CARVALHO, J. J. La etnomusicología en tiempos de canibalismo musical. Una reflexión a partir de las tradiciones musicales afroamericanas. Revista Transcultural de Música. 2003. Disponible en:http://www.redalyc.org/articulo.oa?id=82200705. Acceso en: 8 mayo 2018.

CIRIO, P. Del sueño de la Argentina blancaeuropea a la realidad de la Argentina americana: la asunción del componente étnico-cultural afro y su (nuestro) patrimonio musical. Revista Quilombo, $2007 . \quad$ Disponible en: http://www.revistaquilombo.com.ar/documentos/cirioargentina.pdf. Acceso en: 6 set. 2012. 
Prácticas musicales de procedencia afro en el culto a San Baltazar. La "charanda" de Empedrado (provincia de Corrientes, Argentina). Revista Musical Chilena, Año LVI, $\mathrm{N}^{\circ}$ 197, pp. 9-38, 2002.

CITRO, S. Cuerpos Significantes. Travesías de una etnografía dialéctica. Biblos: Buenos Aires, 2009, p. 351.

CITRO, S.; BROGUET, J.; RODRÍGUEZ, M. y TORRES AGÜERO, S. Performances indígenas y afrodescendientes en Argentina: Recreaciones sonoro-corporales de lo "ancestral, Boletín del Museo Chileno de Arte Precolombino, en prensa.

CUNIN, E. (dir.) Mestizaje, diferencia y nación: Lo "negro" en América Central y el Caribe. México: Centro de estudios mexicanos y centroamericanos, 2010, p.177.

DOMÍNGUEZ, M. E. O 'afro' entre os imigrantes em Buenos Aires: reflexões sobre as diferenças. 2004. 122 f. Tesis (Maestria en Antropologia), Programa de Pós-Graduação em Antropologia Social, Universidade Federal de Santa Catarina, Florianópolis.

ESPINOSA, C. Candombes como memorias vivas. Reflexiones en torno a africanidades en la provincia de Salta, Argentina. Humanidades Populares; Vol. 6; n 8; Primera época, pp. 4660, 2016.

FERNÁNDEZ BRAVO, N. “¿Qué hacemos con los afrodescendientes?” Aportes para una crítica de las políticas de la identidad. In: GUZMÁN, F. y GELER, L. (eds) Cartografías afrolatinoamericanas. Buenos Aires: Editorial Biblos, 2013, pp. 241-262.

FRIGERIO, A. Expansión de religiones afrobrasileñas en Argentina: Representaciones conflictivas de Cultura, Raza y Nación en un contexto de Integración Regional. Archives de Sciences Sociales des Religions (117), pp. 127-150, 2002.

FRIGERIO, A. y LAMBORGHINI, E. "Encontrarse, compartir, resistir: Una "nueva construcción" del candombe (afro) uruguayo en Buenos Aires". Anuario de Antropología Social y Cultural en Uruguay, vol. ${ }^{\circ} 10$, pp. 95-113, 2012.

GARGUIN, E. 'Los Argentinos Descendemos de los Barcos': The Racial Articulation of Middle Class Identity in Argentina (1920-1960). Latin American and Caribbean Ethnic Studies, 2: 2, pp. 161-184, 2007.

GELER, L. ¿Quién no ha sido negro en su vida? Performances de negritud en el carnaval porteño de fin de siglo (xix-xx). In: GARCÍA JORDÁN, P. El Estado en América Latina: recursos e imaginarios. Barcelona: Publicacions i Edicions de la Universitat de Barcelona, 2011, pp. 183-211.

Categorías raciales en Buenos Aires. Negritud, blanquitud, afrodescendencia y mestizaje en la blanca ciudad capital. Runa vol.37 no.1, pp 71-87, 2016.

GUZMÁN, F., GELER, L. y LAMBORGHINI, E. Los estudios afrodescendientes en argentina: nuevas perspectivas y desafíos en un país «sin razas». Tabula Rasa ${ }^{\circ} .27$, pp. 67 $101,2017$.

HALL, S. ¿Quién necesita identidad? In: HALL, Stuart y DU GAY, Paul (comps.). Cuestiones de identidad cultural. Buenos Aires: Amorrortu Editores, 2011[1996], p. 314. 

LITORAL ARGENTINO

LAMBORGHINI, E. Candombe afro-uruguayo en Buenos Aires: Nuevas formas de sociabilidad, política y apropiación del espacio público. 2015. 268 f. Tesis (Doctorado en Antropología), Facultad de Filosofía y Letras, Universidad Nacional de Buenos Aires.

MARTINS, L. Performances do tempo espiralar. In: RAVETTI, G. y ARBEX, M. (orgs.) Performance, exílio, fronteiras - errâncias territoriais e textuais. Belo Horizonte: Departamento de Letras Romanicas, 2002, pp. 69-91.

La oralitura de la memoria. In: PÉREZ-WILKE, I. y MÁRQUEZ, F. Nuestra América Negra: Territorios y voces de la interculturalidad afrodescendiente. Caracas: Universidad Bolivariana de Venezuela, 2013, pp. 17-44.

OCORO LOANGO, A. Afroargentinidad y memoria histórica: la negritud en los actos escolares del 25 de mayo. Propuesta Educativa n 35, Vol 1, pp 127-130, 2011.

PARODY, V. Música, política y etnicidad: convergencias entre democracia y dictadura en el proceso de relocalización del candombe afrouruguayo en Buenos Aires (1973-2013). Resonancias vol. 18, n³4, pp. 127-153, 2014.

REID ANDREWS, G. Recordando África al inventar Uruguay: sociedades de negros en el carnaval del Montevideo, 1865-1930. Revista de Estudios Sociales 26, pp 86-104, 2006.

RESTREPO, E. Identidades: planteamientos teóricos y sugerencias metodológicas para su estudio Revista JANGWA PANA No 5, pp 24-35, 2007.

ROCKWELL, E. La apropiación un proceso entre muchos que ocurren en ámbitos escolares. Anuario de la Sociedad Mexicana de Historia de la Educación nº 1, pp. 28-37, 2005.

SANSONE, L. Negritude sem etnicidade: o local e o global nas relações raciais e na produção cultural negra do Brasil. Salvador: Edufba-Pallas, 2003, p.335.

SEGATO, R. Santos e daimones: o politeismo afrobrasileiro e a tradiçao arquetipal. Brasilia: Editora Universidade de Brasilia, 2005, p. 515.

. La Nación y sus otros. Buenos Aires: Prometeo Libros, 2007, p.350.

SILVA, T. (org.) Identidade e diferença: a perspectiva dos estudos culturais. Petrópolis: Vozes, 2000.

TRIGO, A. Candombe and the reterritorialization of culture. Callaloo, Vol. 16, n³, pp. 716$728,1993$.

VVAA (Red Conceptualismos del Sur) Perder la forma humana. Buenos Aires, EDUNTREF, 2014, p.443.

WADE, P. Música, Raza y Nación. Música tropical en Colombia. Bogotá: Vicepresidencia de la República de Colombia, 2002, p. 409.

\section{Notas}

i Aunque rara vez sea parte del lenguaje nativo, la elección por el término "candombe afro-uruguayo" se debe a la necesidad de hacer dos distinciones, con incidencias en las modalidades de apropiación de la práctica en Argentina. Por un lado, resaltar su pertenencia nacional, pues aquí se relocaliza desde Uruguay -más concretamente desde su capital, Montevideo-, diferenciándose así de un candombe "argentino" que ha sido poco investigado y que durante la última década se ha revisibilizado en el espacio público. Por otro, enfatizar la dimensión étnico-racial -a partir del prefijo "afro"-. Pues su condición de práctica etnicizada/racializada como 
"negra" ha sido una de las más resaltadas por sus practicantes argentinos, a despecho de otras posibles -como las apropiaciones que hizo del candombe la clase obrera migrante europea, por ejemplo (REID ANDREWS, 2010, p.93).

ii Se trata de una denominación etic. A lo largo del trabajo ahondo en las dificultades que presenta esta clasificación en el cotidiano de las personas en el contexto argentino. De hecho, el fragmento de conversación escogido para el título pretende subrayar que la blanquitud funciona en un plano ideal que poco se adecua la experiencia, en ese sentido es que, como señala un practicante, "se desfasa". Agradezco las observaciones de Manuela Rodríguez, en particular en lo que refiere a dar más precisiones sobre el modo en que se presentan las autoidentificaciones de clase y raza en el campo.

iii Rara vez un practicante de candombe se define abiertamente como de "clase media". En todo caso pondrá el acento en "formas de ser", experiencias de la ciudad, trayectorias por circuitos culturales distintas, respecto a las de un candombero afrouruguayo. Aunque en esta oportunidad no ahondaré en la dimensión de clase asociada al eje raza-nación, es necesario mencionar que, como advirtió Garguin (2007) durante el siglo XX, en particular durante el peronismo, esta narrativa de blanquitud fue articulándose en clave racial con una identidad de "clase media", oponiéndose a la de "clase trabajadora". De tal modo se contrapuso la blanquitud y europeidad de esa emergente identidad de clase asumida por sectores medios, a la piel oscura de los "cabecitas negras" - muchos de ellos migrantes de provincias argentinas que constituían las mayorías peronistas - (op.cit.). Lo cual, según lo que vengo investigando, no descarta, por un lado, que estos sectores medios no se hubieran visto incluidos en procesos de mestizaje. Y, por otro, que la posibilidad de movilidad social ascendente y la inclusión en esta emergente identidad de clase media, no supusiera atravesar procesos de blanqueamiento social.

${ }^{\text {iv }}$ He registrado la aparición de categorizaciones raciales cuando una persona asume, para sí o para otro, que el color de piel dice algo acerca de las conductas morales o habilidades sociales. Cuando alguien ve a una persona no-blanca y constata, casi de manera automática, lo que es (o no) capaz de hacer. Operan entonces categorías raciales cuando se cree que los "negros" saben "naturalmente" tocar el tambor y bailar "con gracia y sensualidad". O que los "blancos" no pueden hacerlo. También hay "raza" en los modos en que la persona "racializada" asume (o no) esas asignaciones sociales que les son hechas a partir de características corporales propias, reconocidas por otros a "simple vista". Es decir, si entendemos que las identidades son relacionales, las identificaciones raciales (entre otras) ponen en juego, al mismo tiempo y en diferentes proporciones, "prácticas de asignación e identificación" (RESTREPO, 2007, p.28) como las descriptas.

v Esta necesidad de pensar las continuidades entre la narrativa nacional y los vínculos cotidianos (o lo que él llama "niveles micro y macro") fue advertida por Frigerio (2006) para el caso argentino.

vi Estas formaciones administran ciertas jerarquizaciones socioculturales en base a marcas particulares que permiten identificar tipos de otros internos (indígenas, afrodescendientes, migrantes, etc.) y así regulan condiciones de existencia diferenciales para los mismos, quienes "se reconocen como formando parte histórica o reciente de la sociedad sobre la cual un determinado Estado-Nación extiende su soberanía" (BRIONES, 2008, p.16).

vii Esta idea fue sugerida por Domínguez (2004) para la ciudad de Buenos Aires. Allí se integran prácticas como el candombe afrouruguayo, pero también la capoeira o las danzas afro brasileras, danzas afro-peruanas, etc.

viii Los datos que daré a continuación remiten exclusivamente a las capitales de ambos países, si bien existen diferencias regionales al interior de cada país, ellas han sido los centros de producción y legitimación de las identidades nacionales dominantes, consolidando las versiones más difundidas sobre las trayectorias de la población negra en cada lugar.

${ }^{\text {ix }}$ Se dispone aún de escasa información sobre la actividad de los afroporteños, aunque hay registros de que al menos hasta 1978, en el Shimmy Club -entidad fundada en 1882 por un afroporteño -, se practicaba un candombe local (FRIGERIO, 1993).

${ }^{\mathrm{x}}$ Hecho que en las últimas décadas se ve reforzado por políticas nacionales y transnacionales, como lo fueron la creación del Día del Candombe el 3 de diciembre; y la decisión de la UNESCO, del 2009, de declararlo Patrimonio Inmaterial de la Humanidad.

xi La "llamada" era un procedimiento tradicional que se utilizaba, primero en las Salas de Nación - éstas eran recintos en donde los amos permitían que se agruparan los africanos según su supuesto lugar de procedencia, duraron casi todo el siglo XIX, y fijaron entre sus objetivos la defensa de los intereses de la comunidad -; luego 
en las Comparsas, para comunicarse entre sí valiéndose del lenguaje tímbrico del tambor. En 1956, se oficializa el "Desfile de Llamadas", un desfile competitivo de las asociaciones carnavalescas afrouruguayas.

xii En el Proyecto de Investigación y Desarrollo (PID) "Crisis y subjetividad. Prácticas de matriz afro en la ciudad de Rosario", indagamos en las conexiones y singularidades (estas últimas, derivadas de las diferentes trayectorias históricas de cada una de estas prácticas) de las modalidades de apropiación de estas prácticas.

xiii Segato (2007, p.261) sugiere que, vía expresiones populares negras de países limítrofes, en Argentina se (re) introduce una "cultura de la negritud" que había sido negada y reprimida.

xiv Entre comillas retomo algunas descripciones de mis interlocutores. Otros sectores sociales produjeron en un periodo similar, formas reivindicativas de "lo negro" con otras características y sujetas a otros constreñimientos (ver ADAMOSVSKY, 2012).

xv Me refiero al Barrio "del Tambor" de Paraná (que nucleó a buena parte de la población negra hasta mediados de siglo XIX y se cuenta con registros de sus ejecuciones públicas de "candombes"). Así como a la presencia de la Sociedad Coral Carnavalesca "Negros Santafesinos", compuesta mayormente por afrodescendientes, que salió en los carnavales de Santa Fe hasta 1950 aproximadamente.

xvi Lo cual también diferencia en el sur de esta región de lo que sucede algo más al norte, a la altura de las ciudades de Resistencia (Chaco) y Corrientes (Corrientes). Allí también se iniciaron procesos de reivindicación de un legado africano, pero ligado al culto a San Baltazar, que está vigente en la zona (CIRIO, 2002).

xvii Extraído del cd interactivo Tangó de San Miguel, Paraná, 2008.

xviii Más de una vez, personas que no practican candombe, u otras manifestaciones culturales afroamericanas, me han interrogado como investigadora y practicante, en tono jocoso y dando por obvia cierta incompatibilidad, sobre las motivaciones que llevarían a personas "blancas" a interesarse por estas prácticas "negras". En muchas ocasiones, algunos de estos observadores externos, e incluso algunos investigadores, reducen este interés a un "snobismo". Sin desconocer cierta "moda", intentaré demostrar que, al menos en el contexto analizado, ésta sería una explicación muy simplificadora.

xix El autor especifica que se refiere a Buenos Aires. Sin embargo, teniendo en cuenta que éste $-\mathrm{y}$ otros investigadores- reconocen que la capital porteña "funciona como referencia de la nación en su conjunto" (GELER, 2016, p.74), asumiré su análisis para pensar las realidades de las ciudades del Litoral aquí investigadas. ${ }^{\mathrm{xx}}$ La autora subraya cómo de acuerdo a esta ideología nacional "los afroargentinos habrían desaparecido en parte debido al mestizaje, es decir, se habrían vuelto blancos por dilución/absorción” (GELER, 2016, p.76).

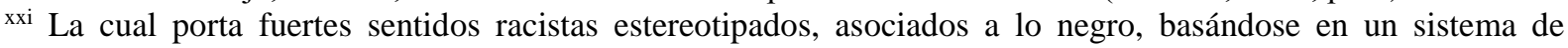
categorización visual según el cual se consideran "negros" exclusivamente a aquellos que respondan a la figura prototípica de la "raza negra" (negrura de la piel, pelo "mota", nariz ancha y labios gruesos).

xxii Si la capital porteña funciona como síntesis de la nación argentina, el "interior" -tal es el apelativo con el cual en Argentina se reduce al resto de las provincias argentinas respecto al "centro" representado por Buenos Aireses la "cara vergonzante" de la misma (BRIONES, 2008, p.28).

xxiii Daré un ejemplo de estos posibles puntos de sutura en el último apartado, cuando me refiera a los señalamientos que muchos practicantes argentinos de candombe afrouruguayo realizan de su color de piel "oscuro" como un indicio de no-blancura, en un juego irregular de espejos entre las propias narrativas familiares y nacionales. La "oscuridad" funciona así como marca de negritud "silenciada" u "omitida" -en el sentido amplio que puede adquirir en Argentina, que subsume todo lo no-blanco (mestizo, indígena y negro)-.

xxiv En la elaboración de esta idea fue sugerente la distinción de Frigerio (2006) entre negros y "negros", el análisis de Geler (2011) sobre las performances de negritud en los carnavales de finales de siglo XIX y comienzos del XX en la capital porteña y las contribuciones de Wade (2002) sobre las vinculaciones entre negritud, performance y cuerpo.

xxv En un artículo planteamos que, en la región, se habían asociado al candombe sentidos "racializados" (vinculados a lo que de negro tiene la práctica), que se relacionaban con lo comunitario, la resistencia, y el vínculo arte-vida; los cuales se vincularon substancialmente a "un modo" propio (y ya no "ajeno") de ejecutar la práctica.

xxvi Más de una vez distintos practicantes de candombe establecieron paralelismos entre las operatorias políticas y subjetivas de olvido, negación y silencio sobre hechos del pasado argentino cercano, con otros del pasado lejano. Por ejemplo, conectando los procesos de recuperación de la identidad de hijas e hijos de desaparecidos durante la última dictadura con la reemergencia de identidades "negras", frente al resquebrajamiento de la versión escolar de la "desaparición" de esta población en el país.

xxvii El "candombe canción" es realizado, sobre todo, como práctica musical individual o con una pequeña

agrupación, en la cual se utilizan otros tambores -en general tumbadoras-.

xxviii Como ha sido observado por Carvalho (2003) para el caso brasilero, por ejemplo. 
xxix Para esta investigación es necesario considerar esos modos diferenciales de regular la existencia de lo negro en Uruguay y Argentina y su incidencia en la visibilidad/invisibilidad de las prácticas culturales (como el candombe) de africanos y afrodescendientes de uno y otro lado. Desde allí es preciso entender cómo hoy se producen las modalidades de apropiación y experiencias de negritud en torno al candombe afrouruguayo en el Litoral argentino.

xxx Me inspiro aquí en observaciones de Moreno Figueroa (en CUNIN, 2010) quien en su abordaje del racismo en México apunta a investigar entre aquel "grupo de población que se posiciona a sí mismo como punto de referencia para hablar del otro [afromexicano y/o indígena] (...) aquel que debido a tal posición no es cuestionado ni problematizado suficientemente" (Op. cit., p.67). En mi caso, me interesa en particular indagar entre aquellas personas que en general asumen la procedencia europea como equivalente de "argentinidad" y la blanquitud algo que se infiere de la misma, aunque no necesariamente se nombre o asuma de manera explícita.

xxxi De allí que se trate de procesos culturales de "desestabilización, recombinación, hibridación y de "cortar y mezclar" (HALL, 2010, p.311). En tanto acontecimiento histórico forzado, es necesario explorar más allá de los intentos de darle una "coherencia imaginaria" a esta experiencia (2010, p.351).

xxxii Me he valido de la noción de gesto performativo de Martins (2002) para comprender el carácter de memoria viva y productiva de estos "modos de hacer". Una memoria, como sugiere la autora "fracturada por la desterritorialización del cuerpo/corpus africano" (MARTINS, 2013, p.37).

xxxiii En un sentido amplio que en Argentina comprende relaciones entre dos tipos de negritud: racial y popular (GELER, 2016) y que por lo tanto no se restringe a africanos y afrodescendientes sino que puede alojar todo lo que se considera "no-blanco": afrodescendiente, indígena y/o mestizo.

${ }^{\text {xxxiv }}$ Frigerio (2006) observa las continuidades que existen entre la negritud racial y popular. El autor sugiere, y aquí asumo esta propuesta, que la población subalterna afroargentina del Buenos Aires de fines del siglo XIX y principios del XX fue "un modelo privilegiado de construcción de [los] sectores populares argentinos" en periodos posteriores. Y la persistencia del término (negro) funciona como un primer indicio.

xxxv Según lo etnografiado, un mismo sujeto puede identificarse como "blanco" o "no-negro", respecto a un "negro de raza negra"; como "negro" en tanto condición de clase reforzada por categorizaciones raciales y como "no-tan-blanco" (u "oscuro", también puede aparecer el término local "morocho"), respecto al otro extremo del continuum racial que crea sujetos "blancos" puros en oposición a otros sujetos "negros" que también lo serían.

xxxvi En este trabajo recuperé también algunos relatos que conectaban el proceso de blanqueamiento de estas memorias familiares a experiencias de movilidad social ascendente y/o de inserción en ciertos circuitos sociales de "clase media", lo cual nos convoca a seguir profundizando en las relaciones entre clase y raza como marcadores de diferencia social que en nuestro contexto interactúan permanentemente en la compleja construcción de lo que es "negro" y lo que es "blanco".

xxxvii "Normalizar" en el sentido de elegir arbitrariamente una identidad específica como el parámetro en relación al cual las otras identidades solo pueden ser evaluadas de forma negativa (Silva, 2000).

xxxviii Frigerio (2006) ha caracterizado a la raza como un elemento "ocluido" de los análisis sobre desigualdad social en Argentina, a favor de la categoría de clase.

xxxix Punto sobre el cual avancé en otro trabajo (BROGUET, 2014).

${ }^{\mathrm{xl}}$ Resignifico aquí en una expresión que aparece en Rinaudo (en Cunin 2010).

\section{Sobre la autora}

Julia Broguet. Becaria del Consejo Nacional de Investigaciones Científicas y Técnicas. Candidata al Doctorado en Antropología de la Universidad de Buenos Aires. Adscripta en "Sistemas Socioculturales Americanos" en la Facultad de Humanidades y Artes de la Universidad Nacional de Rosario. En esta misma casa de estudios integra 
también el Área de Antropología del Cuerpo. Sus investigaciones se especializan en la intersección de esta área con los estudios afrolatinoamericanos/argentinos. Indagó en procesos de racialización (en sus articulaciones con otros marcadores de diferencia social) entre practicantes de manifestaciones culturales "negras" y en la emergencia de articulaciones identitarias en torno a la afrodescendencia en el Litoral argentino. Correo electrónico: lajuliche@ hotmail.com

Recebido: 16.05 .18

Aprovado: 06.06.18 